\title{
The state of neonatal abstinence syndrome in West Virginia
}

\author{
Sean Loudin, MD ${ }^{1}$
}

Author affiliations are listed at the end of this article.

Correspondence to: Sean Loudin, MD Marshall University Joan C. Edwards School of Medicine loudin8@marshall.edu

\section{KEYWORDS}

Neonatal Abstinence Syndrome, Addiction, Opioid

The opioid epidemic has ravaged Appalachia. West Virginia is a state of hard-working individuals, many with blue collar jobs. As people sought relief from the pain they were facing performing their normal daily activities, they turned to opioid medications for relief. An underestimation of the addictive potential of these medicines turned this relief into a catastrophic reality. Gender, race, socioeconomic status and religion offer no protection from the farreaching grasp of addiction. Age also offers no limits to the effects of opioids, with newborns affected by substances they are exposed to while developing in womb.

The brain of a fetus does not discriminate between a prescribed opiate (methadone or buprenorphine) and an illicit one (such as heroin or fentanyl). Exposure results in specific brain adaptations while inside the mother. When the baby is born, the infant is no longer exposed to medications and in essence "quits cold turkey". This results in a flood of adrenaline sweeping through the body. Neonatal withdrawal is the inappropriate reaction of the baby's body systems to its own adrenaline release. Symptoms can include irritation of the nervous system (crying, tremors, sneezing), irritation of the gastrointestinal tract (vomiting, diarrhea), and fever.

The approach to caring for drug-exposed infants has changed over time to keep up with both the different substances babies are exposed to an ever-changing evidence-based medicine practices.

It typically takes weeks to treat babies exposed to opiates. Problems multiply when babies are exposed to many different medications and classes of drugs, whether they are prescribed or obtained by other means. Withdrawal becomes more complex in these cases and after dealing with a very acute phase of withdrawal, babies may also have to deal with a sub acute phase that can last for months after discharge. These babies are at risk for neurodevelopmental consequences, behavioural and/or cognitive impairment. It is imperative to identify these children in order to enable them to receive early intervention programs if necessary and allow for early recognition of developing problems as the child grows older.

National research has shown that opioid exposure increased six times between 2000 and 2009. The 
number of babies affected with neonatal abstinence syndrome (NAS) followed this trend and tripled over that time period. The CDC has ranked West Virginia as number one in the country in terms of neonatal abstinence syndrome, with 33.4 per 1000 live births. ${ }^{1}$ This $3.34 \%$ compares to less than 1 percent nationwide. The CDC numbers do not tell the full story. West Virginia recognizes the limitations of using administrative data and through state collaborative projects has been proactive in identifying affected infants $^{2,3}$. More accurate statistics show that 5.2\% of all babies born in the state are diagnosed with neonatal abstinence syndrome.

For every baby diagnosed with NAS there is a mother at one of the many stages in the cycle of recovery. It is important to remember the mother-baby dyad when discussing neonatal abstinence syndrome. There is so much potential for the mother to help the baby and for the baby to help the mother during treatment and recovery.

Preparing for the days, weeks, months and years ahead is necessary for any comprehensive program. There are no guarantees when it comes to the health and well-being of men, women and children affected by substance use disorders, but through education and support the potential for positive outcomes grows.

\section{AUTHOR AFFILIATIONS}

1. Marshall University Joan C. Edwards School of Medicine, Huntington, West Virginia

\section{REFERENCE}

1. Ko JY, Patrick SW, Tong VT, Patel R, Lind JN, Barfield WD. Incidence of neonatal abstinence syndrome - 28 States, 1999-2013. Morb Mortal Wkly Rep. 2016;65:799-802.

2. Loudin S, Murray S, Davies TH, Evans J, and Werthammer J. An atypical withdrawal syndrome in neonates prenatally exposed to gabapentin and opioids. Journal of Pediatrics. 2016

3. Loudin S, Werthammer J, Prunty L, Shapiro Jl, Davies TH. A Management Strategy from the Front Line of the Neonatal Abstinence Syndrome Epidemic. Journal of Perinatology 2016. 